\title{
Fluid Flow In 2-D Single Phase Petroleum Reservoir
}

\author{
Thamir Abdul Hafedh \\ Computer Sciences Department/College of Education \\ University of Mosul
}

Received

Accepted

1/8/2006

14/11/2006

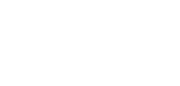

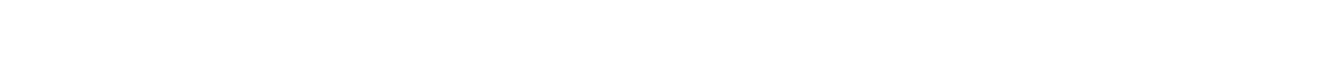

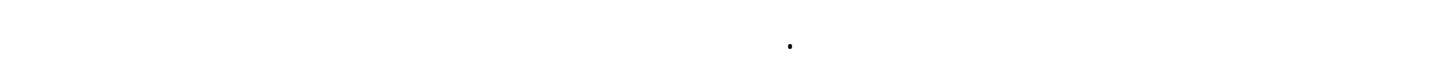

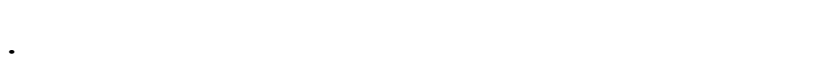

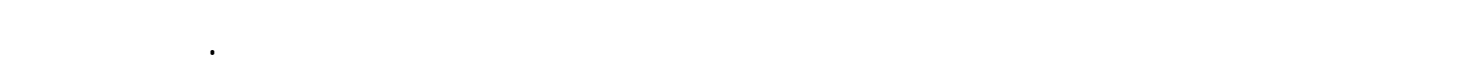

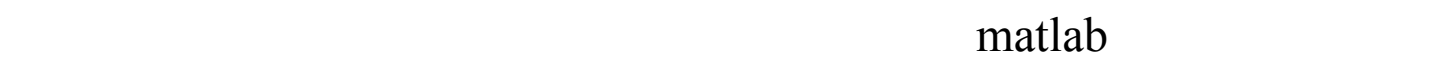

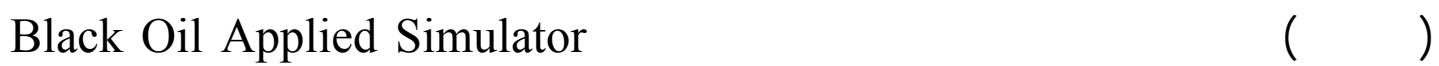

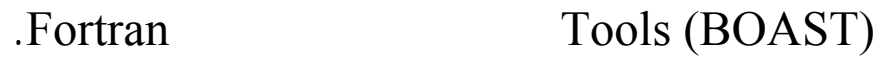

\section{Abstract}

The purpose of this paper is to test the Darcy's equation and to investigate, by simulations how it's suitable for use in single-phase oil reservoir, and to be used later in history matching procedure.

The model used is a flow in a saturated reservoir and this model similar to steady state two-dimensional (2-D) saturated porous media.

Reservoir engineering is based on the understanding of fluid flow in porous media. our aim is to produce a reliable code in Matlab which is relevant for the reservoir process (as simulator), and to be used instead of traditional simulator, Black Oil Applied Simulator Tools (BOAST) which was written in fortran, and widely used in almost works concern reservoir simulation.

Key words: Partial Differential Equation, Finite Difference,

\section{Introduction}

\section{Reservoir, Simulation, Matlab.}

Reservoir engineering is based on the understanding of fluid flow in porous media. We must have some data about permeability, porosity, saturation, and relative permeability for oil. We use a fluid flow model in 2-D flow in a porous media. In this model we consider a flow in a saturated reservoir and it is similar to steady state 2-D saturated porous media. The reservoir flow uses a potential formulation to reservoir management (Landa, et al, 2000). 
The reservoir modeling is complex, multidisciplinary task. Once satisfactorily accomplished, the resulting model is used by operators and other interested parties for predicting performance under the range of operating and maintenance scenarios, for planning development strategies and for assisting production operations. (Parish, et al., 1993)

The first approach, commonly known as geometrical reservoir characterization focuses on static data. This approach uses the spatial correlation of static data to predict the unknown parameters at unsampled locations. In the second approach, subsurface properties are estimated through an inverse modeling procedure, which matches the dynamic data, by comparing simulated production or pressure to field data. We note that the inverse modeling takes into account the fluid flow when assessing reservoir properties. Until now both approaches were used only for predicting geological models, while little attention was given to other reservoir engineering parameters. Moreover, geostatistical methods becomes a major research area after the mid 1980's. Consequently, research in inverse modeling and automatic history matching lost in late 1970 's and early 1980's vigor with few exceptions. Although a number of algorithms have been proposed before the mid 1980's, automatic history matching has not found widespread use yet. A major reason was the use of deterministic optimization methods, which can handle a limited number of parameters.

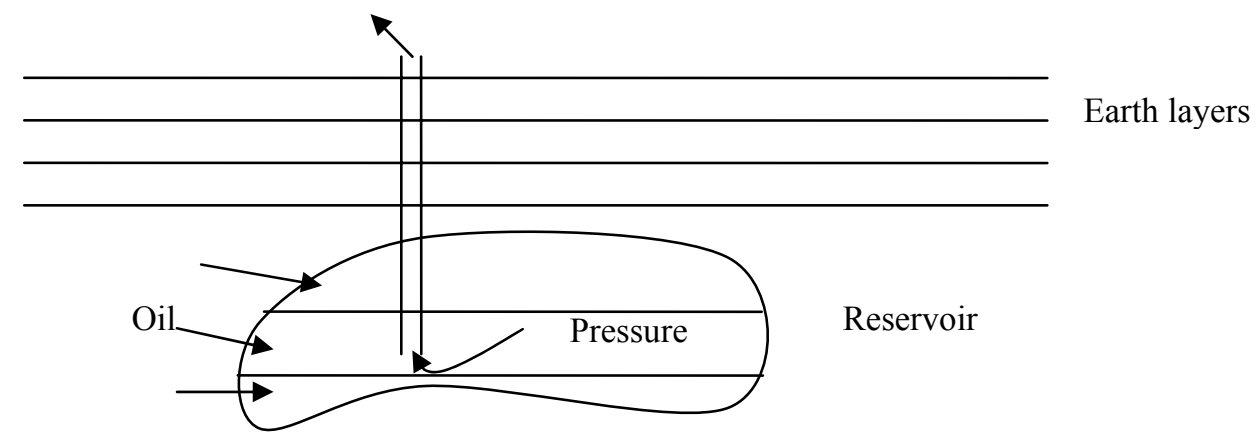

Figure 1. Reservoir description

\section{Previous Works}

History matching is formulated depending on the process of determining unknown parameter values for a mathematical reservoir model, such as permeability and porosity, which give the closest fit of measured and calculated pressures. In principle, one would like an automatic routine for history matching, applicable to simulators of varying complexity, one that does not achieve a set of parameter estimates, Thamir (2005).

In the seventh decade of last century, various automatic and semiautomatic history matching techniques have been introduced. Jacquard and Jain (1965) presented a technique based on a version of the 
method of steepest descent, where they did not consider their method to be fully operational, however, due to the lack of experiences with convergence. Jahns (1965) presented a method based on the GaussNewton equation with a stepwise solution for speeding the convergence; but his procedure still required a large number of reservoir simulation to reach to a solution. Coats et al. (1970) presented a workable automatic history matching procedure based on least-squares and linear programming. Slater and Durrer (1971) presented a method based on a gradient method and linear programming. In their study they mentioned the difficulty of choosing a step size for their gradient method, especially for problems involving low values of porosity and permeability and they also pointed out the need for a fairly small range in their reservoir description parameters for highly non-linear problems.

Thomas et al. (1972) presented a non-linear optimization technique that automatically varies reservoir performance, their method based on the classical Gauss-Newton least-squares procedure, the method is a nonlinear algorithm that will match both linear and non-linear systems in reasonable number of simulations. Wasserman et al. (1975) applied the material presented by two groups of scientists Chen et al. (1974) and Chavent et al. (1975) to practical reservoir problems. The pressure history matching algorithm used was initially based on a discretized single-phase reservoir model. Multiphase effects are approximately treated in the single-phase model by multiplying the transmissibility and storage terms by saturation-simulator run. Thus, all the history matching is performed by a "pseduo" single-phase model. The multiplicative factors for transmissibility and storage are updated when necessary. The matching technique can change some of the properties of the reservoir model.

Lee et al. (1986) presented an algorithm for an automatic history matching which is developed from spline approximations of permeability and porosity distributions and from theory of regularization to estimate permeability or porosity in a 1-Phase, 2-D areal reservoir from well pressure data. The algorithm uses conjugate gradient method as its core minimization method. A number of numerical data are carried out to evaluate the performance of the algorithm. Comparisons with conventional (non-regularized) automatic history matching algorithms indicate the superiority of the new algorithm with respect to the parameter estimates obtained.

\section{Application to a Saturated 2-D 1-Phase Reservoir}

Consider a saturated reservoir which is to have at least one well. Assumed the region is in the $x y$-plane and that the oil moves towards the well in such a way that the velocity vector is in the $x y$-plane. At the top and bottom of the $x y$ region we will assume there is no flow through these 
boundaries. However, assume there is a wide supply from the left and right boundaries so that the pressure is fixed. The problem is to determine the oil flow rates of well, location of well and number of wells so that there is still oil to be pumped out.

If a cell does not contain a well, then $u_{x}+v_{y}=0$. If there is a well in a cell, then $u_{x}+v_{y}<0$. The motion of the fluid is governed by an empirical law which is analogous to the Fourier heat law.

where

$$
\text { Darcy's Law. }(u, v)=-K\left(h_{x}, h_{y}\right)
$$

$h$ is the hydraulic head pressure and

$K$ is the hydraulic conductivity which is constant for saturated regions.

So, we have $u_{x}+v_{y}=-\left(K h_{x}\right)_{x}-\left(K h_{y}\right)_{y}$ is zero or negative.

NO FLOW THROUGH THIS SIDE

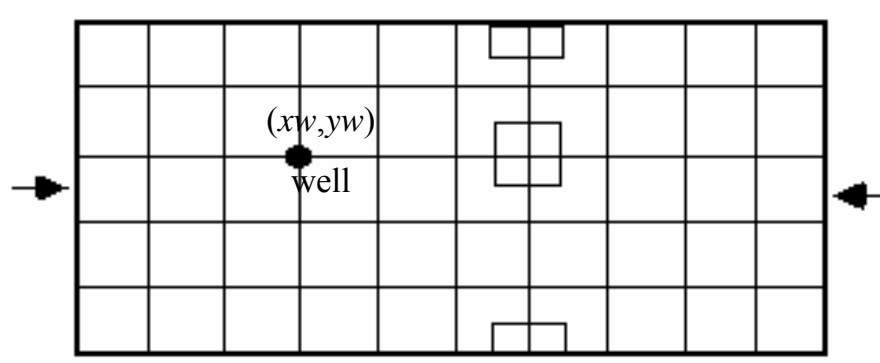

NO FLOW THROUGH THIS SIDE

Figure 2. 2-D 1-Phase reservoir flow in porous media.

\section{Model}

The model has a partial differential equation similar to that of the 2-D heat diffusion model, with have different boundary conditions. For fluid flow reservoir problems, they are either a given function along part of the boundary, or they are a zero derivative for the remainder of the boundary.

\section{Fluid Flow Reservoir Model}

$$
\begin{gathered}
-\left(K h_{x}\right)_{x}-\left(K h_{y}\right)_{y}=\left\{\begin{array}{cc}
0 & ,(x, y) \notin \text { well } \\
-R, & (x, y) \in \text { well }
\end{array}(x, y) \in(0, L) \times(0, H), \mathrm{R}>0 .\right. \\
K h_{y}=0 \text { for } \mathrm{y}=0 \text { and } y=H, \text { and } \\
h_{x}=h_{0} \text { for } x=0 \text { and } x=L .
\end{gathered}
$$




\section{Problem Treatment}

In this problem the finite difference method used with the SOR iterative method, for the $(d x d y)$ cells in the interior. For the portions of the boundary where the derivative is set equal zero on a half cell $(d x / 2 d y)$ or $(d x d y / 2)$, some additional code inserted inside the SOR loop, for example, the model where $h_{y}=0$ at $y=H$ on the half cell $(d x d y / 2)$. The finite difference equation and the corresponding line of SOR code are, respectively, $u=h$ :

$-[(u(i+1, j)-u(i, j)) / d x-(u(i, j)-u(i-1, j)) / d x] / d x-[(0)-(u(i, j)-u(i, j-1)) / d y] /(d y / 2)=0$

utemp $=\left((u(i+1, j)+u(i-1, j)) /\left(d x^{*} d x\right)+2 * u(i, j-1) /\left(d y^{*} d y\right)\right) /\left(2 /\left(d x^{*} d x\right)+2 /\left(d y^{*} d y\right)\right)$.

$u(i, j)=(1-w)^{*} u(i, j)+w^{*} u t e m p$.

In the following implementations observe that the extra lines of code are that reflect these derivative boundary conditions.

\section{Implementation.}

The fluid flow reservoir model uses the following parameters:

$$
\begin{array}{llll}
L=5,000 & d x=h=100 & x w=(i w-1) h & h \infty=100 \\
H=1,000 & d y=h=100 & y w=(j w-1) h & K=10 .
\end{array}
$$

A single well with a flow rate of -1000 was used in the first numerical experiment. The first output graph plots of the hydraulic head pressure as a function of $x$ and $y$. Note that the pressure near the well has dropped from 100 to about 30. The second experiment has two wells with the same flow rate and in this case the pressures are negative near both wells, this indicates that before any steady state solution was achieved, the wells become dry!

Matlab Code for Fluid Flow in 2-D 1-Phase Saturated Reservoir in Porous Media.

clear all

$\mathrm{K}=10$;

well=-1000;

iw $=16$;

$\mathrm{jw}=6$;

eps $=0.0001$;

$\mathrm{nx}=50$;

ny $=10$;

$\mathrm{H}=1000$;

$\mathrm{w}=1.7$;

$\mathrm{u}=$ ones $(\mathrm{nx}+1, \mathrm{ny}+1) * 100$;

$\mathrm{h}=\mathrm{H} / \mathrm{ny}$;

$\operatorname{maxit}=400$;

tol=eps*h*h;

for $\mathrm{m}=1$ : maxit

numi $=0$;

$\mathrm{j}=1$;

for $\mathrm{i}=2$ :nx 


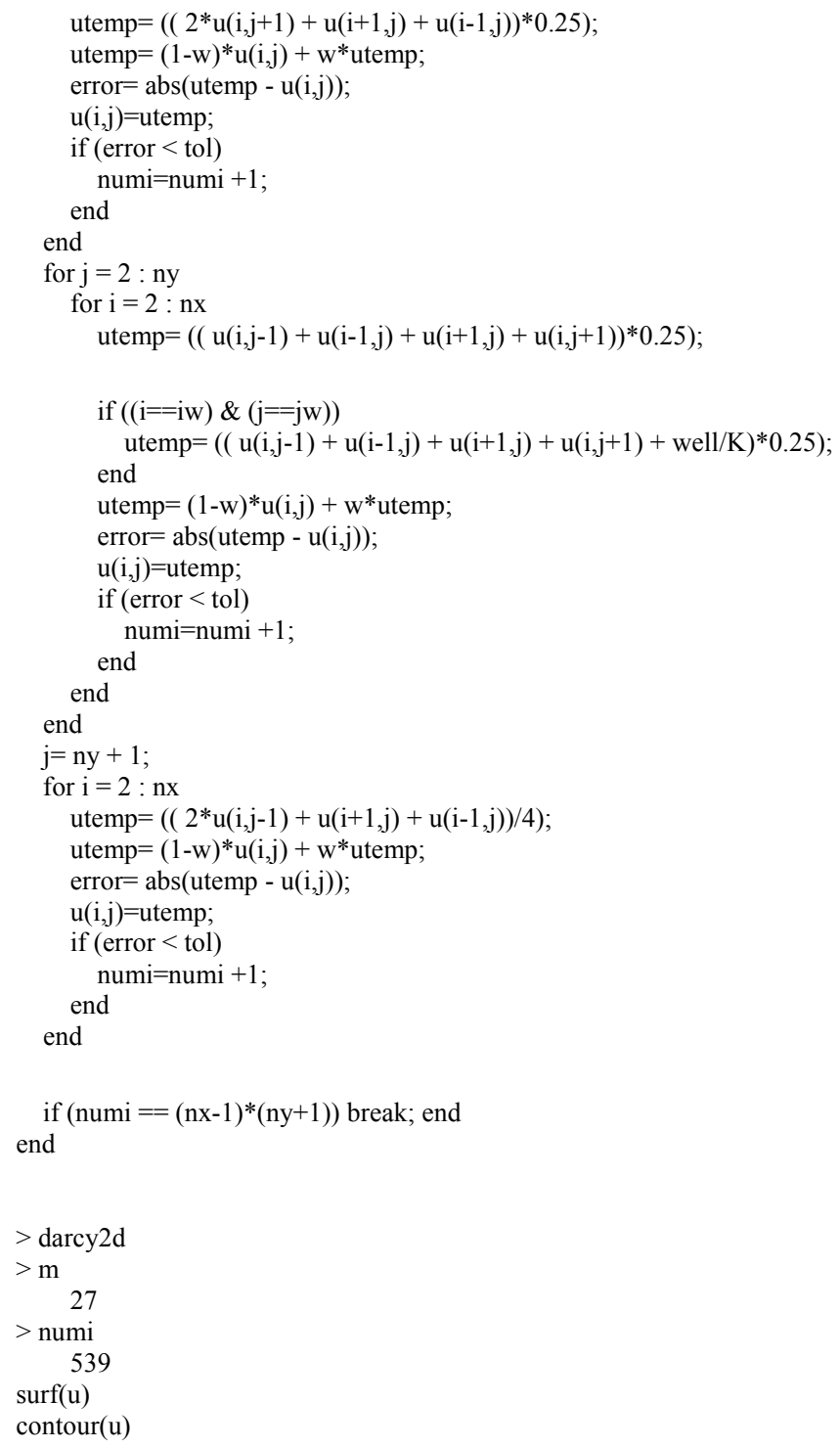

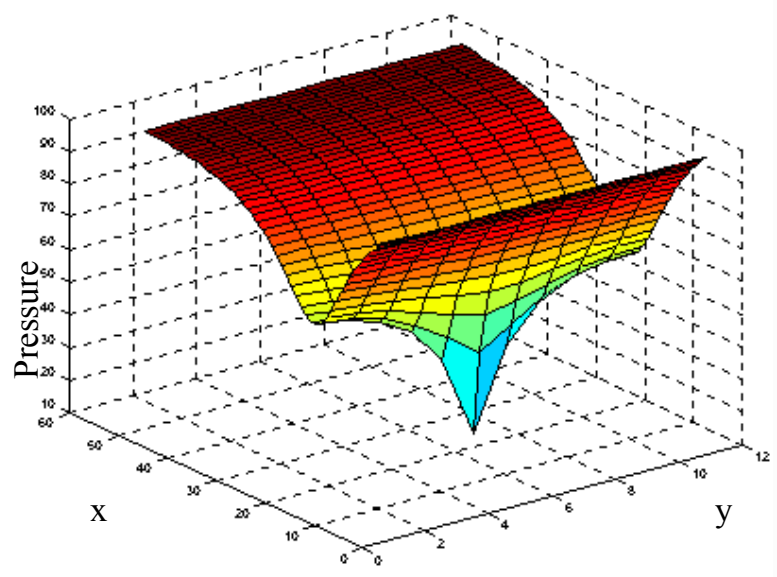

a. Surf 3-D

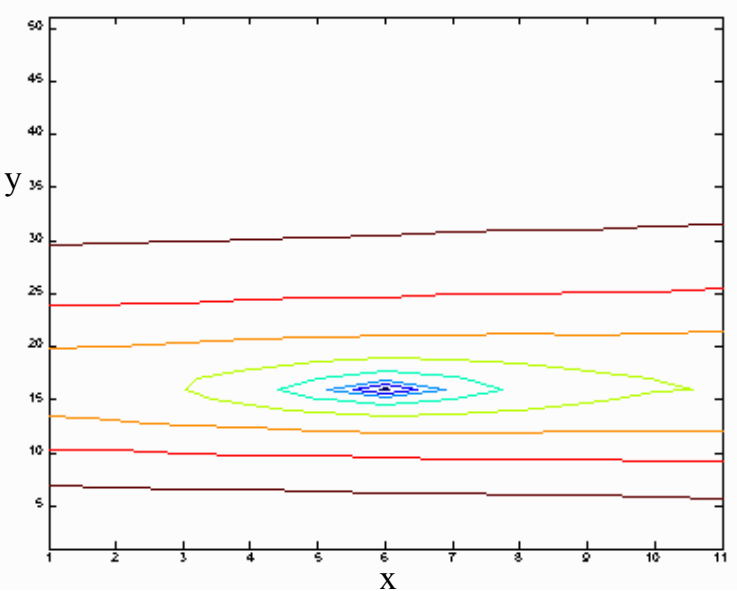

b. Contour 2-D

Figure 3. Well at $(16,6)$ with flow Rate 1000. 


\section{Results Analysis}

A two-dimensional, black-oil reservoir considered as shown in Figure 3. In this instance, to avoid the effects of the heterogeneity in understanding the problem from the conceptual point of view, the permeability and porosity were set constant throughout the reservoir. In this model, well $(16,6)$ is producing at constant flow rates of 1000 . Figure 4 shows that the pressure in the reservoir is a function of time if we have two wells production $(16,6)$ and $(36,4)$ with flow rates of 1000 . The pressure maps are shown here to illustrate what is going on in the reservoir and to help the understanding the results of the analysis.

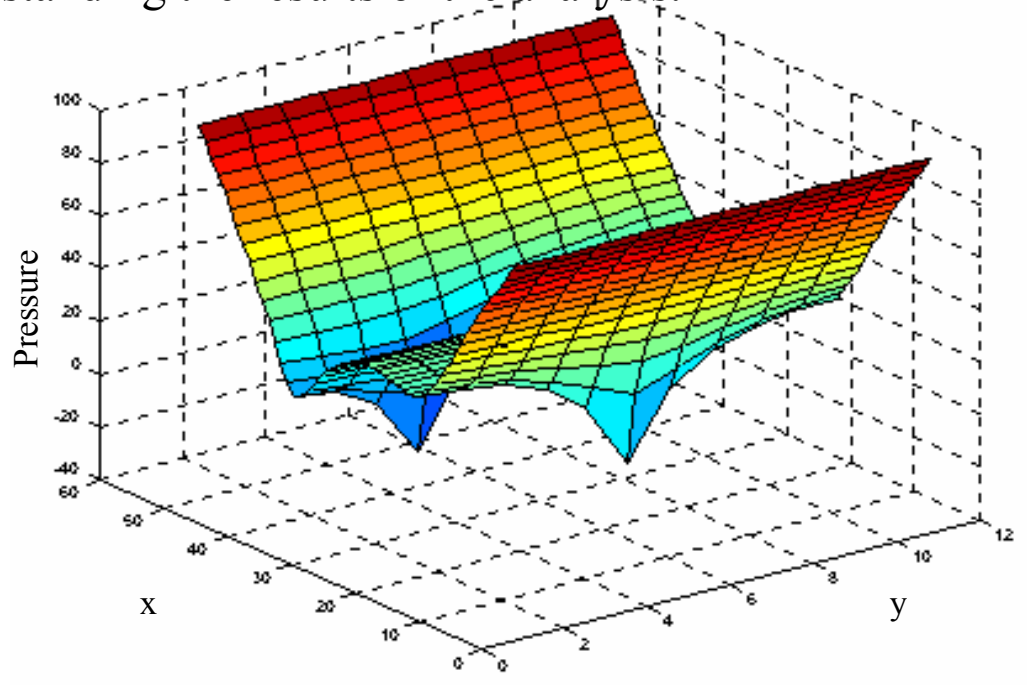

a. Surf 3-D

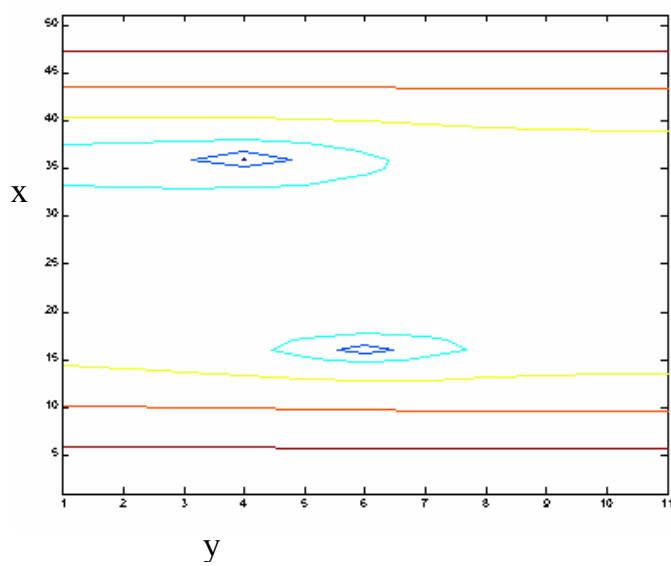

b. Contour 2-D

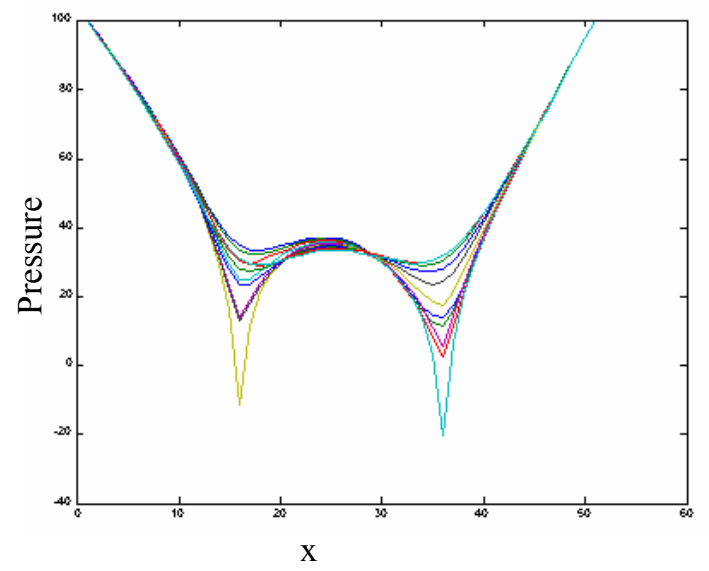

c. Plot 2-D

Figure 4. Wells at $(16,6)$ and $(36,4)$ with flow rates of 1000.

\section{Conclusions}

The first objective which is the main purpose of this work is to develop the procedure of Darcy's equation for fluid flow. The second objective is to develop a new procedure to be used as a simulator of reservoir to assess the reservoir behavior. 


\section{References}

Chavent, G., Dupuy, M., and Lemonnier, P., 1975; "History-Matching by Use of Optimal Control Theory", SPE Journal, 259, 74-86.

Chen, W. H., Gavalas, G. R., Seinfeld, J. H., and Wasserman, M. L., 1974; "A New Algorithm for Automatic History Matching", SPE Journal, 257, 593-608.

Coats, K. H., Dempesy, J. R., and Henderson, J. H.; 1970, “A new Technique for Determining Reservoir Description from Field Performance Data", SPE Journal, 249, 66-74.

Jacquard, P., and Jain, C.; 1965, "Permeability Distributions from Field Pressure Data", SPE Journal, 234, 281-294.

Jahns, H. O.; 1965, "A Rapid Method for Obtaining a Pressure Response Data", SPE Journal, 237, 315-327.

Landa, J. L, Horne R.N, M.M. Kamal, and C.D. Jenkins, 2000, Reservoir Characterization Constrained to Well-Test Data: A Field Example, Arco Exploration and Production Technology

Lee, T., Kavaris, C., and Seinfeld, J. H., 1986; "History-Matching by Spline Approximation and Regularization in Single-Phase Areal Reservoirs", SPE Reservoir Engineering Journal, Sept. 521-543.

Parish, R. G., Calderbank, V. J., Watkins, A. J., Muggeridge, A. H., Goode, A. T., and Robinson, P. R., 1993; "Effective HistoryMatching: The Application of Advanced Software Techniques to the History-Matching Process", SPE Technical Papers 25250.

Slater, G. E., and Durrer, E. J.; 1971, "Adjustment of Reservoir Simulation Models to Match Field Performance", SPE Journal, 251, 259-305.

Thamir, A. Hafedh, 2005," Modified Algorithm For Automatic HistoryMatching By Use of Optimal Control Theory" Dissertation, Doctor of Philosophy, Gadjah Mada University, Yogyakarta, Indonesia.

Thomas, L. K., Hellums, L. J., and Reheis, G. M.; 1972, “A Nonlinear Automatic History Matching Technique for Reservoir Simulation Model", SPE Journal, 253.

Wasserman, M. L., Emanuel, A. S., and Seinfeld, J. H., 1975; "Practical Applications of Optimal Control Theory to History-Matching Multiphase Simulator Reservoir Models", SPE Journal, 259, 347355. 